\title{
Interfacial, electrical, and spin-injection properties of epitaxial Co2MnGa grown on
} GaAs(100)

Damsgaard, Christian Danvad; Hickey, M. C.; Holmes, S. N.; Feidenhans, R.; Mariager, S. O.; Jacobsen, C. S.; Hansen, J.B.

Published in:

Journal of Applied Physics

Link to article, DOI:

$10.1063 / 1.3148298$

Publication date:

2009

Document Version

Publisher's PDF, also known as Version of record

Link back to DTU Orbit

Citation (APA):

Damsgaard, C. D., Hickey, M. C., Holmes, S. N., Feidenhans, R., Mariager, S. O., Jacobsen, C. S., \& Hansen, J. B. (2009). Interfacial, electrical, and spin-injection properties of epitaxial Co2MnGa grown on GaAs(100). Journal of Applied Physics, 105(12), 124502. https://doi.org/10.1063/1.3148298

\section{General rights}

Copyright and moral rights for the publications made accessible in the public portal are retained by the authors and/or other copyright owners and it is a condition of accessing publications that users recognise and abide by the legal requirements associated with these rights.

- Users may download and print one copy of any publication from the public portal for the purpose of private study or research.

- You may not further distribute the material or use it for any profit-making activity or commercial gain

- You may freely distribute the URL identifying the publication in the public portal 


\title{
Interfacial, electrical, and spin-injection properties of epitaxial $\mathrm{Co}_{2} \mathrm{MnGa}$ grown on $\mathrm{GaAs}(100)$
}

\author{
C. D. Damsgaard, ${ }^{1,2, a)}$ M. C. Hickey, ${ }^{3, b)}$ S. N. Holmes, ${ }^{2, c)}$ R. Feidenhans'l, ${ }^{4}$ S. O. Mariager, ${ }^{4}$ \\ C. S. Jacobsen, ${ }^{1}$ and J. B. Hansen ${ }^{1}$ \\ ${ }_{1}^{1}$ Department of Physics, Technical University of Denmark, DK-2800, Lyngby, Denmark \\ ${ }^{2}$ Cambridge Research Laboratory, Toshiba Research Europe Limited, 208 Cambridge Science Park, Milton \\ Road, Cambridge, CB4 OGZ, United Kingdom \\ ${ }^{3}$ Department of Physics, Cavendish Laboratory, University of Cambridge, J. J. Thomson Avenue, \\ Cambridge, CB3 OHE, United Kingdom \\ ${ }^{4}$ Niels Bohr Institute, Nano-Science Center, University of Copenhagen, 2100 Copenhagen Ø, Denmark
}

(Received 30 December 2008; accepted 10 May 2009; published online 16 June 2009)

\begin{abstract}
The interfacial, electrical, and magnetic properties of the Heusler alloy $\mathrm{Co}_{2} \mathrm{MnGa}$ grown epitaxially on $\operatorname{GaAs}(100)$ are presented with an emphasis on the use of this metal-semiconductor combination for a device that operates on the principles of spin-injection between the two materials. Through systematic growth optimization the stoichiometry in the bulk $\mathrm{Co}_{2} \mathrm{MnGa}$ can be controlled to better than $\pm 2 \%$, although the interface is disordered and limits the spin-injection efficiency in a practical spintronic device irrespective of the half-metallic nature of the bulk metal. Molecular beam epitaxial growth was monitored in situ by reflection high energy electron diffraction and the bulk composition was measured ex situ with inductively coupled plasma optical emission spectroscopy. The $\mathrm{Co}_{2} \mathrm{MnGa}$ $L 2_{1}$ cubic structure is strained below a thickness of $20 \mathrm{~nm}$ on GaAs(100) but relaxed in films thicker than $20 \mathrm{~nm}$. Electrical measurements on the $\mathrm{Co}_{2} \mathrm{MnGa}$ reveal general characteristics of a disordered electron system with insulating behavior for layer thicknesses $<4 \mathrm{~nm}$. Thicker layers show a negative magnetoresistance with extraordinary Hall effect constants up to $30 \Omega \mathrm{T}^{-1}$. Spin polarization transfer across the interface between $\mathrm{Co}_{2} \mathrm{MnGa}$ and $\mathrm{GaAs}$ is approximately $6.4 \%$ at 5 $\mathrm{K}$ in the current of a GaAs $p$ - $i-n$ diode even with compositional disorder at the interface. (c) 2009 American Institute of Physics. [DOI: 10.1063/1.3148298]
\end{abstract}

\section{INTRODUCTION}

The spin-transmission efficiency at the ferromagnetic metal-semiconductor interface is seen as one of the most important parameters in establishing the viability of hybrid spintronic devices. Indeed, the International Technology Roadmap for Semiconductors ${ }^{1}$ refers specifically to the generic Heusler alloy, GaAs combination, as an emerging research material if the challenges presented by interface stability, stoichiometric control, and spin transmission can be overcome. In future practical computing devices spininjection and extraction are the essential mechanisms for readout of the computational state. Interface stability and stoichiometry are important as the half-metallic properties of Heusler alloys are generally lost in atomically disordered systems. A range of full Heusler alloy materials have already been grown epitaxially on GaAs. These include $\mathrm{Ni}_{2} \mathrm{MnGa}$, $\mathrm{Ni}_{2} \mathrm{MnGe}$ and $\mathrm{Ni}_{2} \mathrm{MnAl},{ }^{2} \mathrm{Co}_{2} \mathrm{MnSi},{ }^{3}$ and $\mathrm{Co}_{2} \mathrm{MnGa}$ which were incorporated into a spin-light emitting diode (spinLED) structure. ${ }^{4}$ In fact other candidate Heusler alloy materials have been fabricated into spin-LED devices, including $\mathrm{Co}_{2} \mathrm{MnGe}^{5}$ and the off-stoichiometric $\mathrm{Co}_{2.4} \mathrm{Mn}_{1.6} \mathrm{Ga}^{6}$

\footnotetext{
${ }^{\text {a) }}$ Present address: Department of Micro- and Nanotechnology, Technical University of Denmark, DTU Nanotech, Lyngby, Denmark.

${ }^{b}$ Present address: Francis Bitter Laboratory, MIT, Cambridge MA02139, USA.

${ }^{c)}$ Author to whom correspondence should be addressed. Electronic mail: s.holmes@crl.toshiba.co.uk.
}

In practice none of the Heusler alloys have significantly improved the relatively efficient Fe:AlGaAs spin LED device $^{7}$ to any great extent although theoretically the potential has always been there. An early review ${ }^{8}$ of the halfmetallic properties of materials including Heusler alloys for magnetoresistance devices has been followed by a review ${ }^{9}$ of hybrid devices fabricated by molecular beam epitaxy (MBE). The magnetic and electrical transport properties of the Heusler alloys themselves have an interesting range of behavior from positive and negative magnetoresistance effects ${ }^{10}$ to electrical characteristics of a strongly disordered system. ${ }^{2,3}$ $\mathrm{Co}_{2} \mathrm{MnGa}$ (with bulk lattice constant $5.77 \AA$ ) is closely lattice matched to GaAs (5.65 $\AA$ ), has a controllable dipolar and magnetocrystalline uniaxial magnetic anisotropy ${ }^{11}$ and is predicted $^{12}$ to be close to half-metallic with a small but finite density of states in the minority spin band at the Fermi energy of the system. In the present work we discuss the interface properties, the electron transport behavior in the bulk and the spin-injection efficiency between $\mathrm{Co}_{2} \mathrm{MnGa}$ and a GaAs- $\mathrm{In}_{0.18} \mathrm{Ga}_{0.82} \mathrm{As}$ quantum well spin-LED structure.

\section{STRUCTURAL PROPERTIES AND STOICHIOMETRY OF $\mathrm{CO}_{2} \mathrm{MNGA}$}

The atomic layer control achievable with MBE has enabled the growth of thin layers of the ferromagnetic Heusler alloy $\mathrm{Co}_{2} \mathrm{MnGa}$ on $\mathrm{GaAs}(100)$ substrates. The MBE chamber (VG Special Systems) has a base pressure of 5 $\times 10^{-10}$ mbar with an in situ reflection high energy electron 
TABLE I. The structural details of the MBE grown devices.

\begin{tabular}{ccccccc}
\hline \hline Device & Wafer & Comment & Composition (Co:Mn:Ga) & \multicolumn{3}{c}{$\begin{array}{c}\text { Thickness } \\
(\mathrm{nm})\end{array}$} \\
\hline 1 & VG04-069 & & $1.91: 1.10: 1.00$ & 6.4 & 9.5 & 15.9 \\
2 & VG05-064 & Spin-LED & $2.25: 1.02: 1.00$ & 10 & & \\
3 & VG05-070 & & $2.06: 0.95: 1.00$ & 3.7 & 7.5 & \\
4 & VG05-088 & XMCD data (Ref. 14) & $1.86: 0.99: 1.00$ & 5.3 & 7.6 & 9.7 \\
5 & VG05-089 & XMCD data (Ref. 14) & $1.95: 0.98: 1.00$ & 5.3 & 7.6 & 9.7 \\
6 & VG06-002 & & & 11 & 22 & 44 \\
\hline a Consisting of a GaAs- In $_{0.18} \mathrm{Ga}_{0.82}$ As-GaAs quantum well in a $p-i-n$ GaAs doping structure. &
\end{tabular}

${ }^{\mathrm{a}}$ Consisting of a GaAs-In ${ }_{0.18} \mathrm{Ga}_{0.82} \mathrm{As}-\mathrm{GaAs}$ quantum well in a $p-i-n$ GaAs doping structure.

diffraction system. $\mathrm{Co}_{2} \mathrm{MnGa}$ was grown by co-evaporation from three separate sources consisting of two effusion cells and one electron-gun source. This provided atomic beams of $\mathrm{Co}, \mathrm{Mn}$, and $\mathrm{Ga}$ with the deposition rate monitored by quartz crystal microbalances at each source. The $\mathrm{Co}_{2} \mathrm{MnGa}$ growth rate was $0.5 \mathrm{ML} / \mathrm{min}$ with substrate temperatures up to $250{ }^{\circ} \mathrm{C}$ possible.

The composition of the MBE layers was determined by inductively coupled plasma optical emission spectroscopy after calibration of the technique with bulk $\mathrm{Co}_{2} \mathrm{MnGa}$ alloy. The relative deviation from nominal 2:1:1 composition with the present epitaxial devices is better than $\pm 2 \%$. Energy dispersive spectroscopy based on $\mathrm{x}$-ray florescence analysis was also used to determine the composition, however this technique was found to be less sensitive and large errors could be observed even in a bulk reference material. Table I is a summary of the device thicknesses and compositions.

$\mathrm{X}$-ray diffraction (XRD) was used to investigate the crystal structure of the Heusler thin films and their crystal orientation relative to the $\mathrm{GaAs}(100)$ substrate (both in-plane and out-of-plane orientations). The most detailed data was obtained at the synchrotron beam-line at Hasylab. ${ }^{13}$ Figure 1 shows typical $\mathrm{x}$-ray diffractograms where the scattered $\mathrm{x}$-ray intensity is measured as a function of $k_{\text {perpendicular }}$ (out-of-

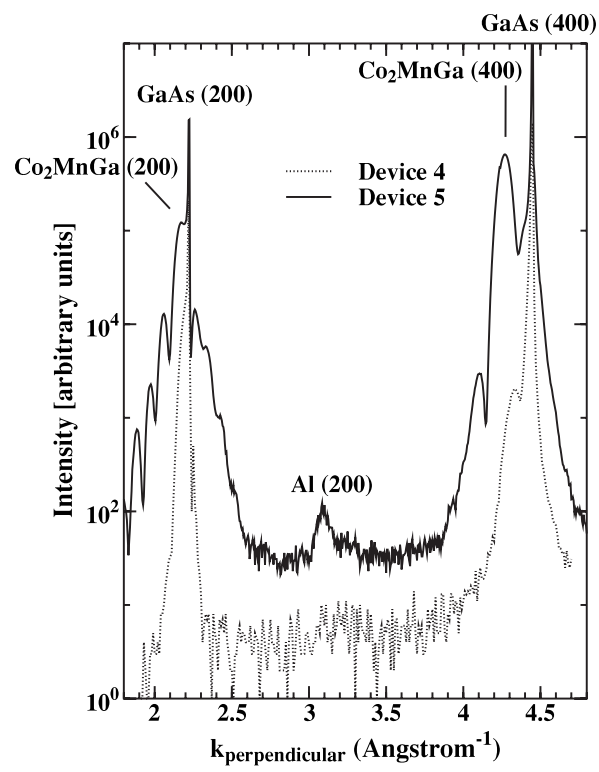

FIG. 1. XRD intensity as a function of in-plane scattering angle for devices 4 and 5. The (200) and (400) order reflections for the $\mathrm{Co}_{2} \mathrm{MnGa}$ epilayer and the GaAs substrate are indicated. A background (200) order reflection from the $\mathrm{Al}$ cap is also indicated. plane scattering wave vector). Devices 4 and 5 with relative cobalt fractions of 1.86 and 1.95 are both $5.3 \mathrm{~nm}$ thick layers of nominally $\mathrm{Co}_{2} \mathrm{MnGa}$ capped with $3 \mathrm{~nm}$ of Al. The (200) and (400) order Bragg reflections are clearly seen for both the GaAs substrate and the $\mathrm{Co}_{2} \mathrm{MnGa}$ epilayers. In addition clear interference fringes are observed that originate from reflections from the top and bottom interfaces of the $\mathrm{Co}_{2} \mathrm{MnGa}$ layer. Anisotropy in the Bragg reflections for parallel and perpendicular substrate orientations show that a strained cubic structure has been stabilized. This demonstrates that $\mathrm{Co}_{2} \mathrm{MnGa}$ grows epitaxially on the $\mathrm{GaAs}(100)$ surface in the strained $L 2_{1}$ cubic structure. The strain increases with the thickness of the $\mathrm{Co}_{2} \mathrm{MnGa}$ layer. For the two $5.3 \mathrm{~nm}$ films in Fig. 1 we find peaks at $k_{\text {perpendicular }}=4.34$ and $4.27\left(\AA^{-1}\right)$ giving an out-of-plane lattice parameter $a=5.79$ and $5.89 \AA$, compared to the GaAs lattice constant $=5.65 \AA$ and bulk $\mathrm{Co}_{2} \mathrm{MnGa}=5.77 \AA$. The $\mathrm{Co}_{2} \mathrm{MnGa}$ thin films are typically strained by up to $2 \%$ (tensile) in the out-of-plane direction and compressed in-plane. The compression inplane is due to the $2.1 \%$ lattice mismatch with GaAs. In thinner layers, in-plane XRD shows that the in-plane strain is not isotropic, the strain being higher along [011] compared to the $[0 \overline{1} 1]$ direction. $\mathrm{Co}_{2} \mathrm{MnGa}$ layers thicker than a critical thickness of $20 \mathrm{~nm}$ are relaxed.

$\mathrm{X}$-ray magnetic circular dichroism (XMCD) measurements were also used to determine the moments on the individual lattice sites. The main observations are a reduction in the predicted magnetic moment on the Mn-lattice site by a factor of five and a total magnetic moment independent of stoichiometry. ${ }^{14}$ The effect of the substrate temperature during growth was also investigated. In situ Auger spectroscopy combined with $\mathrm{Ar}^{+}$sputtering yielded the relative changes of the chemical composition of the Heusler layer and the adjacent layers, i.e., the GaAs substrate and the non-magnetic cap-layer. Intermixing between the Heusler layer and the substrate was negligible up to a substrate temperature of $250{ }^{\circ} \mathrm{C}$. This was also the case with short anneals at $350{ }^{\circ} \mathrm{C}$. However with $450{ }^{\circ} \mathrm{C}$ anneals, Co migrated into the GaAs and other phases formed, particularly $\mathrm{CoGa}_{3}$.

\section{ELECTRICAL PROPERTIES AND MAGNETORESISTANCE}

Hall bars were patterned along the $[0 \overline{1} 1]$ and the $[0 \overline{11}]$ directions using standard optical lithography techniques followed by ion-beam etching. AC excitation currents of 100 nA at $333 \mathrm{~Hz}$ were used with Stanford SR830 lock-in ampli- 


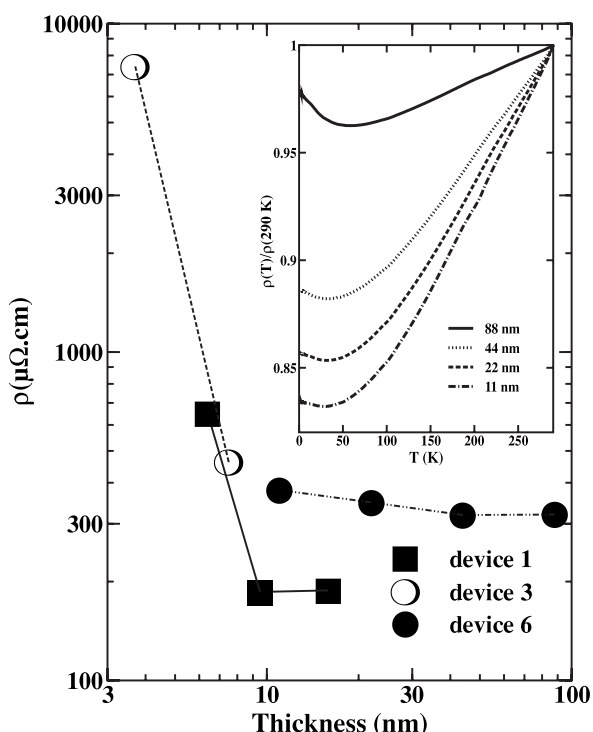

FIG. 2. The resistivity as a function of layer thickness for devices 1,3 , and 6 . The inset shows the temperature dependence of the resistivity for the four thicknesses of device 6 .

fiers. At $300 \mathrm{~K}$ the bulk resistivity of the $\mathrm{Co}_{2} \mathrm{MnGa}$ films was $200-400 \mu \Omega \mathrm{cm}$ for layers with a thickness $(t)>10 \mathrm{~nm}$, this is shown in Fig. 2. In cooling to $30 \mathrm{~K}$ the resistivity decreased by $\sim 5 \%$ showing typical metallic behavior [see Fig. 2 (inset)]. Further cooling to $4 \mathrm{~K}$ resulted in a slight increase of resistivity. This increase in resistance below $30 \mathrm{~K}$ (the actual temperature depends on the layer thickness and can be up to $60 \mathrm{~K}$ ) in device 6 is a result of the strongly disordered nature of the films and is due to weak localization and electron-electron interaction rather than the signature of any spin scattering mechanisms. This behavior has been seen in $\mathrm{Co}_{2} \mathrm{MnSi}^{3}{ }^{3} \mathrm{Ni}_{2} \mathrm{MnGe},{ }^{2}$ and $\mathrm{NiMnSb} .{ }^{15} \mathrm{Co}_{2} \mathrm{MnGa}$ layer thicknesses $<4 \mathrm{~nm}$ were found to be insulating. This may be caused by interfacial disorder or contamination of the interface. Shunting of the current in some of the devices by the $\mathrm{Au}$ or Al capping layer can explain the difference in extrapolated bulk resistivity for $t>10 \mathrm{~nm}$. The resistivities are different in the orthogonal $[0 \overline{1} 1]$ and $[0 \overline{11}]$ directions with the lowest resistivity measured along the [011] direction. This is clearer in the thinner layers, for example in device $6(11 \mathrm{~nm}$ thickness) there is $4.5 \%$ difference in resistivity for the two orthogonal directions, although the size of the negative magnetoresistance is the same in each direction when the difference of background resistivity is taken into account. Differences in resistivity for the thicker layers are within the random experimental error $(<0.9 \%)$ between different Hall bars patterned in the same in-plane direction.

The hard-axis magnetoresistance $\left(\rho_{x x}\right)$ and Hall effect $\left(\rho_{x y}\right)$ of the $\mathrm{Co}_{2} \mathrm{MnGa}$ devices was measured either in a $9 \mathrm{~T}$ or a $12 \mathrm{~T}$ cryostat with base temperatures of $1.7 \mathrm{~K}$. The easy-axis (in-plane) anisotropic magnetoresistance has previously been measured in $\mathrm{Co}_{2} \mathrm{MnGa}^{16}$ Device 1 showed a negative magnetoresistance for all film thicknesses and this is also a characteristic of weak localization and electronelectron interaction effects. A positive magnetoresistance for $B<1 \mathrm{~T}$ is partly due to an in-plane easy-axis switching (with characteristic hysteretic behavior) due to a small in-

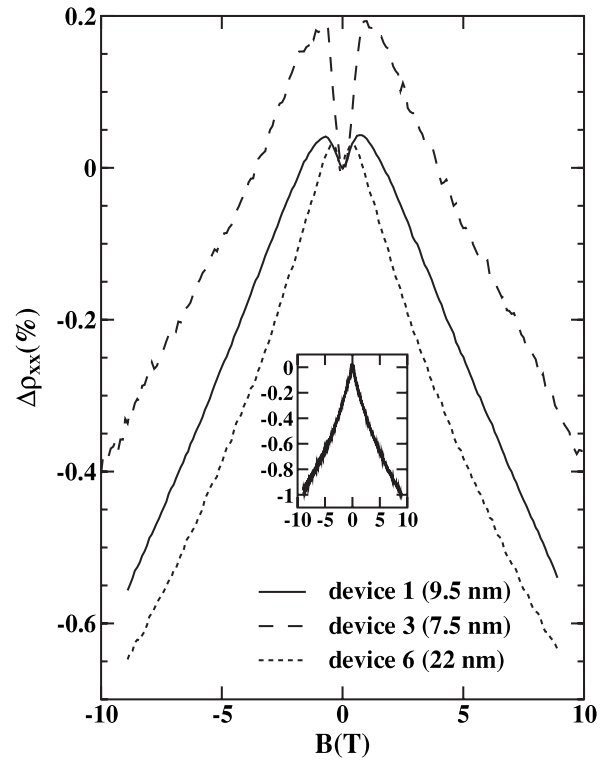

FIG. 3. The magnetoresistance change up to $\pm 9 \mathrm{~T}$ at $1.7 \mathrm{~K}$ for devices 1,3 , and 6 that show a positive magnetoresistance effect at low field and a negative magnetoresistance $>1 \mathrm{~T}$. The inset shows the magnetoresistance percentage change in the $44 \mathrm{~nm}$ thick layer of device 6 .

plane component of the applied perpendicular magnetic field. Figure 3 shows three devices that have positive magnetoresistance behavior at low field. A positive magnetoresistance of $0.19 \%$ up to $1 \mathrm{~T}$ is observed in device 3 before a negative magnetoresistance of $0.6 \%$ at $10 \mathrm{~T}$. All devices show a negative magnetoresistance once any positive effect has been saturated. The inset of Fig. 3 shows the magnetoresistance in the $44 \mathrm{~nm}$ thick layer of device 6 . The $1 \%$ negative magnetoresistance at $9 \mathrm{~T}$ is a general feature of this Heusler alloy, although in this particular device there is no positive magnetoresistance at low magnetic field. This could be due to the larger thickness in device 6 that shows stronger characteristics of bulk-like transport behavior. The same behavior has been reported previously ${ }^{2}$ in other ferromagnetic Heusler alloys, albeit with high in-plane magnetic fields.

The devices all show ordinary and extraordinary Hall effect characteristics, with $\rho_{x y}$ given by

$$
\rho_{x y}(B)=R_{O} B+R_{E} M(B),
$$

where $R_{0}$ is ordinary Hall constant $(=1 / n e$, where $n$ is the electron density), $R_{E}$ is the extraordinary Hall constant, and $M(B)$ is the magnetic field (B) dependent magnetization. The lithographic definition of the potential arms of the Hall bar is such that a Hall resistance in the 1-5 $\Omega$ range is well defined with a Hall voltage of 0 (ignoring the hysteresis inherent in $M$ ) at zero applied field and no intermixing of the longitudinal resistivity component which is typically $1000 \Omega$. A sheet carrier density is measured in the Hall effect, and thin layers were measured here to increase the relative size of the Hall voltage for a given carrier density. The extraordinary Hall effect dominates $\rho_{x y}$ at low magnetic field as the normal Hall constant is typically only 20 to $40 \mathrm{~m} \Omega \mathrm{T}^{-1}$ due to the metallic carrier density and can only be determined accurately when the magnetization has saturated. Figure 4 shows the Hall resistance at $1.7 \mathrm{~K}$ for device 6 up to \pm 9 T. The hysteretic extraordinary Hall resistance 


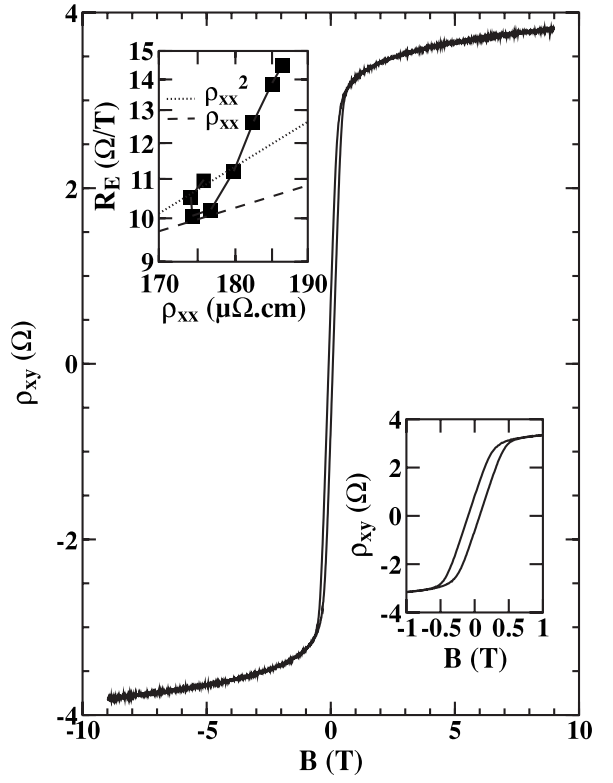

FIG. 4. The Hall resistivity up to $\pm 9 \mathrm{~T}$ at $1.7 \mathrm{~K}$ showing both the normal and the extraordinary Hall effects for the $44 \mathrm{~nm}$ thick layer of device 6 . The insets show the hysteretic behavior of the extraordinary Hall effect up to \pm 1 $\mathrm{T}$ and the extraordinary Hall constant as a function of the (temperature dependent) resistivity.

(see the lower inset to Fig. 4) saturates with the magnetization vector in applied fields $>\sim 2 \mathrm{~T}$. Although it is unclear what the accurate carrier density is in $\mathrm{Co}_{2} \mathrm{MnGa}$ as $d \rho_{x y} / d B$ is still nonlinear at $12 \mathrm{~T}$, the lower limit to the carrier density from $\rho_{x y}$ at $12 \mathrm{~T}$ is $3.8 \times 10^{22} \mathrm{~cm}^{-3}$. In comparison, for the case of the dilute magnetic semiconductor (DMS) GaMnAs, the maximum free hole carrier density is in the region of 1 to $9 \times 10^{20} \mathrm{~cm}^{-3}$ but this depends on the Mn concentration and the level of activation of the Mn dopant. ${ }^{17}$ In the $9.5 \mathrm{~nm}$ thick layer of device $1, R_{E}$ (determined from $d \rho_{x y} / d B$ ) is $14.5 \Omega \mathrm{T}^{-1}$ at $300 \mathrm{~K}$ and $11 \Omega \mathrm{T}^{-1}$ at $1.7 \mathrm{~K}$. This can be seen in the upper inset to Fig. 4 which shows $R_{E}$ as a function of the zero field resistivity. $\rho_{x x}$ has a significant weak localization contribution at $50 \mathrm{~K}$ (where $\rho_{x x}$ is in the region of $175 \mu \Omega \mathrm{cm}$ ), this additional correction to $\rho_{x x}$ would have to be quantified before the dependence of $R_{E}$ on $\rho_{x x}$ could be determined. In this range of temperature (300-1.7 K) $R_{E}$ does not scale with $\rho_{x x}$ or $\rho_{x x}$ (Ref. 2) (see the upper inset in Fig. 4). This behavior is characteristic of a complex dependence of the extraordinary Hall effect on side-jump and/or skew-scattering that has been observed in the DMS GaMnAs, ${ }^{17}$ although the carrier density in $\mathrm{Co}_{2} \mathrm{MnGa}(>3.8$ $\left.\times 10^{22} \mathrm{~cm}^{-3}\right)$ is much higher than in GaMnAs $\left(\sim 10^{20}\right.$ $\left.-10^{21} \mathrm{~cm}^{-3}\right)$. The largest extraordinary Hall constant is $30 \Omega \mathrm{T}^{-1}$ in device 6 for the $22 \mathrm{~nm}$ thick layer and $15 \Omega \mathrm{T}^{-1}$ in the $11 \mathrm{~nm}$ layer. This reduction in $R_{E}$ is a further indication that interface effects are beginning to dominate the electrical properties at thickness $<20 \mathrm{~nm}$ in $\mathrm{Co}_{2} \mathrm{MnGa}$.

\section{SPIN INJECTION}

The efficiency of electron spin-polarization transmission across the $\mathrm{Co}_{2} \mathrm{MnGa}-\mathrm{GaAs}$ interface was determined from a spin-LED structure. ${ }^{18}$ This structure (device 2 in Table I)

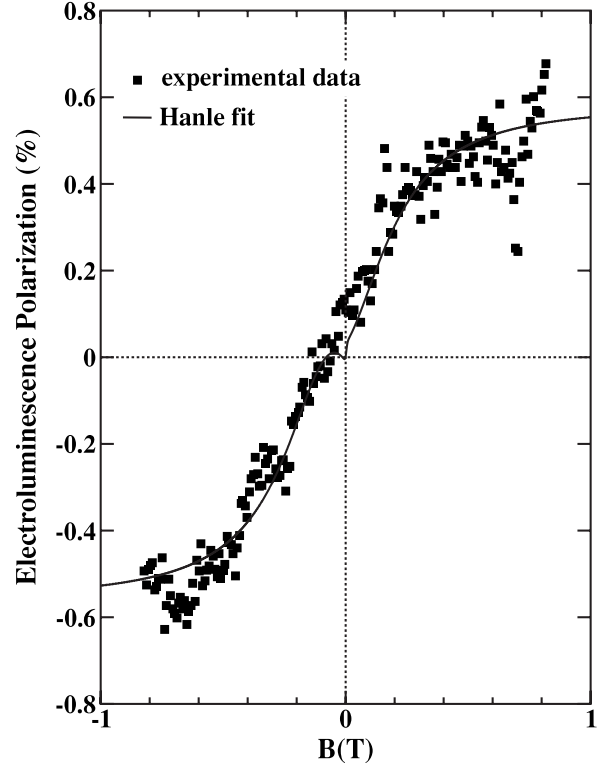

FIG. 5. The electroluminescence polarization from the spin-LED as a function of applied magnetic field in the Hanle geometry. The device was at $5 \mathrm{~K}$ with a current density of $0.2 \mathrm{~A} \mathrm{~cm}^{-2}$. The Hanle angle $\varphi$ is $60^{\circ}$ and $T_{s}$ $=75$ ps from the Hanle fit.

consisted of a $10 \mathrm{~nm} \operatorname{In}_{0.18} \mathrm{Ga}_{0.82}$ As quantum well in the intrinsic region of a GaAs $p-i-n$ doping structure. A surface Schottky barrier of Si doped GaAs at $5 \times 10^{18} \mathrm{~cm}^{-3}$ ensured that an efficient tunneling contact ${ }^{19}$ was made and that the effects of the $\mathrm{Co}_{2} \mathrm{MnGa}$ : GaAs interface could be quantified. Previous spin-LED devices with $\mathrm{Co}_{2.4} \mathrm{Mn}_{1.6} \mathrm{Ga}$, have had a surface Schottky barrier of doped $\mathrm{Al}_{0.2} \mathrm{Ga}_{0.8} \mathrm{As}$ (Ref. 6) or $\mathrm{Al}_{0.33} \mathrm{Ga}_{0.67} \mathrm{As}$ (Ref. 4) in the case of $\mathrm{Co}_{2} \mathrm{MnGa}$. The currentvoltage characteristics of the spin-LED are diode-like with apparent resistances at low currents $(10 \mu \mathrm{A})$ in forward bias on the order of $1-300 \Omega \mathrm{mm}^{2}$. Current is injected between the $\mathrm{Co}_{2} \mathrm{MnGa}$ Schottky contact and a $p$-type Ohmic contact to the GaAs $p$-doped layer. Although an epitaxial Fe reference contact had the same contact resistance on the spinLED, in other structures (a delta-doped GaAs substrate), $\mathrm{Co}_{2} \mathrm{MnGa}$ had two orders of magnitude higher contact resistance compared to epitaxial Fe suggesting strong levels of disorder (or contamination) at the interface between $\mathrm{Co}_{2} \mathrm{MnGa}$ and $\mathrm{GaAs}$.

The oblique Hanle geometry ${ }^{20}$ was used for spinpolarization measurements with the exciton emission energy at $1.329 \mathrm{eV}$ from the $\operatorname{In}_{0.18} \mathrm{Ga}_{0.82}$ As quantum well. Figure 5 shows the electroluminescence polarization signal at 1.329 $\mathrm{eV}$ with a current density of $0.2 \mathrm{~A} \mathrm{~cm}^{-2}$, as a function of applied magnetic field at $5 \mathrm{~K}$. The Hanle angle $(\varphi)$, i.e., the angle between the applied magnetic field and the surface normal is $60^{\circ}$ and this introduces a geometric factor $\cos (\varphi) \sin (\varphi)$ into the estimation of the electrical spininjection efficiency $(\Pi)$ between the $\mathrm{CO}_{2} \mathrm{MnGa}$ and the $\mathrm{In}_{0.18} \mathrm{Ga}_{0.82}$ As quantum well. The optical polarization signal $\left(P_{\mathrm{EL}}\right)$ shown in Fig. 5 is defined as

$$
P_{\mathrm{EL}}=\frac{I_{\sigma^{+}}-I_{\sigma_{-}}}{I_{\sigma_{+}}+I_{\sigma_{-}}},
$$

where $I_{\sigma+}\left(I_{\sigma-}\right)$ is the measured intensity of right (left) circularly polarized electroluminescence. The Hanle curve fit in 
Fig. 5 is determined in the following way. The steady-state Bloch solution for the spin state $\mathbf{S}$, (with components $S_{x}, S_{y}$, and $S_{z}$ ) in the $\operatorname{In}_{0.18} \mathrm{Ga}_{0.82} \mathrm{As}$ quantum well can be written as

$$
S_{z}=\left(\frac{T_{s}}{\tau}\right) S_{O y} \frac{(B / \Delta B)^{2} \sin (\varphi) \cos (\varphi)}{1+(B / \Delta B)^{2}}
$$

where $T_{s}$ is the spin lifetime, $\tau$ is the radiative lifetime, $\Delta B$ is the Hanle curve width, and $S_{O y}$ is the $y$-component (in-plane) of the $\mathrm{Co}_{2} \mathrm{MnGa}$ magnetization and is given by

$$
S_{O y}=S_{O} \tan h\left[a\left(B-B_{c}\right)\right]
$$

where $a$ is the hardness of the hysteresis loop, $B_{c}$ is the coercive field of the $\mathrm{Co}_{2} \mathrm{MnGa}$, and $S_{O}$ is the initial spin state. This captures the essential features of the Hanle curve close to $B=0$. The existence of a plateau in the theoretical fit indicates a sensitivity of the polarization signal to the reversal of magnetization in the $\mathrm{Co}_{2} \mathrm{MnGa}$ layer itself, although this is not usually observed experimentally due to the noise level. The radiative lifetime of the exciton in the $\mathrm{In}_{0.18} \mathrm{Ga}_{0.82} \mathrm{As}$ quantum well, determined from separate time dependent photoluminescence measurements was 350 ps in an identical (but undoped) version of this device. From the Hanle curve fit in Fig. 5 the effective spin lifetime $\left(T_{s}^{*}\right)$ is 75 ps (assuming an electron $g$ factor of -0.7 ) and this gives an efficiency $\left(T_{s}^{*} / \tau\right)$ of 0.21 for emission from the $\mathrm{In}_{0.18} \mathrm{Ga}_{0.82} \mathrm{As}$ quantum well in this structure. The effective spin lifetime is where radiative recombination of the electron spin is accounted for via $1 / T_{s}^{*}=1 / T_{s}+1 / \tau$. Although the optical polarization signal has a maximum of $0.6 \%$, the extracted electrical spin-injection efficiency $(\Pi)$ at the $\mathrm{Co}_{2} \mathrm{MnGa}: \mathrm{GaAs}$ interface is $6.4 \%$ at $5 \mathrm{~K}$. This is determined from Eq. (3) when the $P_{\mathrm{EL}}$ signal is saturated, i.e.,

$$
\Pi \frac{T_{s}^{*}}{\tau}=\frac{P_{\mathrm{EL}}}{\cos \varphi \sin \varphi},
$$

with the measured $P_{\mathrm{EL}}$ signal according to Eq. (2). The polarization signal approaches $0 \%$ at $20 \mathrm{~K}$ in a similar fashion to the off-stoichiometric $\mathrm{Co}_{2.4} \mathrm{Mn}_{1.6} \mathrm{Ga}$ device ${ }^{6}$ with an $\mathrm{Al}_{0.2} \mathrm{Ga}_{0.8} \mathrm{As}$ surface layer. The lower spin-injection efficiency with $\mathrm{Co}_{2} \mathrm{MnGa}$ compared to $\mathrm{Fe}$ (Ref. 7) is consistent with the higher contact resistance and the subsequent loss of spin-polarization above $20 \mathrm{~K}$. In Fig. 6 we show the optical polarization signal from the Hanle geometry as a function of current density at $5 \mathrm{~K}$ and $0.8 \mathrm{~T}$. This reduction in polarization signal with current density (or applied voltage) is partly due to the spin-orbit coupling strength in the $\operatorname{In}_{0.18} \mathrm{Ga}_{0.82} \mathrm{As}$ quantum well enhancing the D'yakonov-Perel spin relaxation mechanism ${ }^{21}$ but is also characteristic of a system with excess $n$-type carriers. ${ }^{22}$ A steady-state population of electrons in the well reduces the overall spin-polarization of the system and this has been shown to be voltage dependent in the GaAs-AlGaAs spin-LED. ${ }^{22}$ This can complicate the interpretation of the role played by interfaces and spindependent scattering effects in the $n$-type doped regions of the spin-LED.

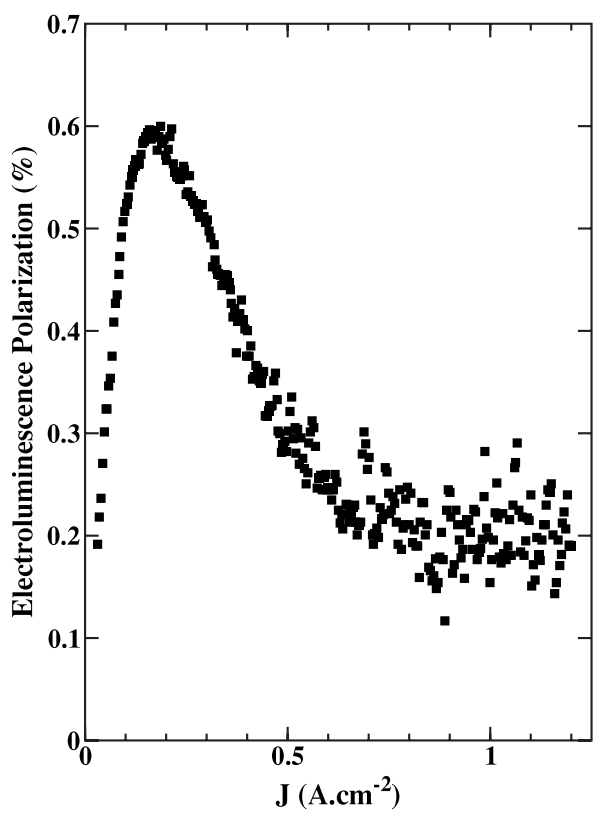

FIG. 6. The electroluminescence polarization in device 2 at $5 \mathrm{~K}$ as a function of current density at $0.8 \mathrm{~T}$ in the Hanle geometry with $\varphi=60^{\circ}$.

\section{CONCLUSIONS}

We have demonstrated that $\mathrm{MBE}$ has the control for the growth of the Heusler alloy $\mathrm{Co}_{2} \mathrm{MnGa}$ on a $\mathrm{GaAs}$ substrate with variations in composition to better than $\pm 2 \%$. A range of surface sensitive techniques has been used to measure the atomic composition, crystal structure of the bulk, and the properties of the interface with GaAs. The electrical and magnetoresistance behavior of bulk $\mathrm{Co}_{2} \mathrm{MnGa}$ is similar to that of a disordered alloy although devices with layer thickness $<4 \mathrm{~nm}$ are insulating. Thicker layers show a negative magnetoresistance effect $(0.7 \%$ at $12 \mathrm{~T})$ with extraordinary Hall effect constants up to $30 \Omega \mathrm{T}^{-1}$ at $0.1 \mathrm{~T}$. Electrical spininjection into a GaAs-based spin-LED with a $10 \mathrm{~nm}$ thick $\mathrm{Co}_{2} \mathrm{MnGa}$ injecting layer is $6.4 \%$ efficient at $5 \mathrm{~K}$, although the spin-polarization signal is reduced to zero above $20 \mathrm{~K}$. Future measurements should address the issue of the temperature dependence of the spin-injection efficiency if the Heusler alloys are to remain as viable emerging research material for practical computing devices.

\section{ACKNOWLEDGMENTS}

We acknowledge Hasylab Hamburg for access to high resolution x-ray diffraction. The GaAs buffer layers and the GaAs- $\mathrm{In}_{0.18} \mathrm{Ga}_{0.82} \mathrm{As}$ quantum well spin LED wafers were grown at the Niels Bohr Institute, Nano-Science Center in the University of Copenhagen by Claus B. Sørensen.

\footnotetext{
${ }^{1}$ http://www.itrs.net/Links/2007ITRS/Home2007.htm

${ }^{2}$ M. S. Lund, J. W. Dong, J. Lu, X. Y. Dong, C. J. Palmstrøm, and C. Leighton, Appl. Phys. Lett. 80, 4798 (2002).

${ }^{3}$ L. J. Singh, Z. H. Barber, Y. Miyoshi, Y. Bugoslavsky, W. R. Branford, and L. F. Cohen, Appl. Phys. Lett. 84, 2367 (2004).

${ }^{4}$ M. C. Hickey, S. N. Holmes, I. Farrer, D. A. Ritchie, M. Pepper, C. D. Damsgaard, C. S. Jacobsen, and J. B. Hansen, Appl. Phys. Lett. 92, 232101 (2008).

${ }^{5}$ X. Y. Dong, C. Adelmann, J. Q. Xie, C. J. Palmstrøm, X. Lou, J. Strand, P. A. Crowell, J. P. Barnes, and A. K. Petford-Long, Appl. Phys. Lett. 86,
} 
102107 (2005).

${ }^{6}$ M. C. Hickey, C. D. Damsgaard, I. Farrer, S. N. Holmes, A. Husmann, J. B. Hansen, C. S. Jacobsen, D. A. Ritchie, R. F. Lee, G. A. C. Jones, and M. Pepper, Appl. Phys. Lett. 86, 252106 (2005).

${ }^{7}$ A. T. Hanbicki, B. T. Jonker, G. Istkos, G. Kioseoglou, and A. Petrou, Appl. Phys. Lett. 80, 1240 (2002).

${ }^{8}$ W. E. Pickett and J. S. Moodera, Phys. Today 54, 39 (2001).

${ }^{9}$ A. Hirohata, A. Kikuchi, N. Tezuka, K. Inomata, J. S. Claydon, Y. B. Xu, and G. van der Laan, Curr. Opin. Solid State Mater. Sci. 10, 93 (2006).

${ }^{10}$ J. Moodera and D. Mootoo, J. Appl. Phys. 76, 6101 (1994).

${ }^{11}$ S. N. Holmes and M. Pepper, J. Supercond. Novel Magn. 16, 191 (2003).

${ }^{12}$ I. Galanakis, P. H. Dederichs, and N. Papanikolaou, Phys. Rev. B 66, 174429 (2002)

${ }^{13}$ Instrument BW2 at HasyLab, Hamburg, http://hasylab.desy.de/

${ }^{14}$ J. S. Claydon, S. Hassan, C. D. Damsgaard, J. B. Hansen, and C. S. Jacobsen, J. Appl. Phys. 101, 09J506 (2007).
${ }^{15}$ W. R. Branford, S. K. Clowes, M. H. Syed, Y. V. Bugoslavsky, S. Gardelis, J. Androulakis, J. Giapintzakis, C. E. A. Grigorescu, S. A. Manea, R. S. Freitas, S. B. Roy, and L. F. Cohen, Phys. Rev. B 69, 201305 (2004).

${ }^{16}$ S. N. Holmes and M. Pepper, Appl. Phys. Lett. 81, 1651 (2002).

${ }^{17}$ K. W. Edmonds, K. Y. Wang, R. P. Campion, A. C. Neumann, C. T. Foxon, B. L. Gallagher, and P. C. Main, Appl. Phys. Lett. 81, 3010 (2002).

${ }^{18}$ The diode mesas were $500 \times 500 \mu \mathrm{m}^{2}$ with $240 \times 240 \mu \mathrm{m}^{2} \mathrm{Co}_{2} \mathrm{MnGa}$ contacts.

${ }^{19}$ O. M. J. van't Erve, G. Kioseoglou, A. T. Hanbicki, C. H. Li, B. T. Jonker, R. Mallory, M. Yasar, and A. Petrou, Appl. Phys. Lett. 84, 4334 (2004).

${ }^{20}$ V. F. Motsnyi, P. van Dorpe, W. van Roy, E. Goovaerts, V. I. Safarov, G. Borghs, and J. De Boeck, Phys. Rev. B 68, 245319 (2003).

${ }^{21}$ I. Zutic, J. Fabian, and S. Das Sarma, Rev. Mod. Phys. 76, 323 (2004).

${ }^{22}$ M. Yasar, R. Mallory, A. Petrou, A. T. Hanbicki, G. Kioseoglou, C. H. Li, O. M. J. van't Erve, and B. T. Jonker, Appl. Phys. Lett. 94, 032102 (2009). 